\title{
BIOREACTOR LANDFILLS: COMPARISON AND KINETICS OF THE DIFFERENT SYSTEMS
}

\author{
Valentina Grossule *, Luca Morello, Raffaello Cossu and Maria Cristina Lavagnolo
}

University of Padova, DICEA, Lungargine Rovetta, Voltabarozzo, 35127 Padova, Italy
Article Info:
Received:
11 June 2018
Revised:
8 August 2018
Accepted:
5 September 2018
Available online:
17 September 2018
Keywords:
Bioreactor landfill
Aerobic landfill
Semi-aerobic landfill
Hybrid landfill
Sustainable landfill

\begin{abstract}
The need for more sustainable landfilling has increased interest in bioreactor landfills as a suitable tool for optimising degradation processes. Bioreactors can be categorised as follows: anaerobic, aerobic, semi aerobic and hybrid. The choice of a specific bioreactor can be strongly influenced by the desired treatment objectives (i.e., energy recovery, increased rate of waste stabilisation, washing) as well as by the specific site conditions (e.g., waste characteristics, climate and social/economic situation, regulations). However, the increased rate of waste stabilisation should be the primary driving principle in the bioreactor landfill design (Cossu, 2010). Full-scale bioreactor landfills are still uncommon and one of the reasons is the perception that the effectiveness of this technology is not well demonstrated. This paper aims to contribute to filling this knowledge gap by analysing and comparing the lab scale applications of different types of bioreactors available in the literature and providing a survey of the different methods by considering their respective advantages and disadvantages. Qualitative analysis of the main types of bioreactor landfills is provided according to a few selected characteristics (i.e. energy recovery, biochemical kinetics, technological complexity, costs). Considering landfill sustainability, the discussion is primarily focused on the quantification of the stabilisation capability of the different bioreactors which is calculated in terms of COD and ammonia removal kinetics. The results demonstrate that the optimisation of COD removal kinetics is the highest in aerated bioreactors, while ammonia removal kinetics is maximum in hybrid bioreactors (i.e., 6 and 10 times higher, respectively, compared to the anaerobic bioreactors).
\end{abstract}

\section{INTRODUCTION}

Although recent legislation tends to limit landfilling as much as possible, it will continue to play a key role in future modern solid waste management systems (Cossu, 2012). Even with circular economy thinking, the zero-waste concept cannot currently be realistically achieved and a final disposal step is needed for residues that cannot be technically or economically exploited. Landfilling assumes the role of providing a final sink to close the loop in the material cycle in order to isolate, from the environment, concentrated residual waste that are no longer usable. In particular sustainable landfilling has been introduced as a system that should be operated in such a way to minimise the emissions potential by achieving waste stabilisation as quickly as possible in order to preserve the next generations from potential environmental risks and remediation costs.

From an environmental and health point of view, the most problematic issue dealt with in a landfill system is the putrescible fraction of waste. This fraction is responsible for the main long-term impacts, including methane and carbon dioxide emissions (contributing to the greenhouse effects and ozone depletion) and leachate emissions resulting in surface and groundwater pollution as well as soil pollution. In order to achieve the sustainability requirements, several strategies can be adopted to control the effects caused by the landfilling of biodegradable waste. These control strategies can be implemented before landfilling by means of the diversion of the putrescible fraction from the waste stream going to the landfill (separate collection), thermal or mechanical/biological pre-treatment and washing of the waste, and during the operational and/or aftercare phases by using in-situ treatments approaches.

Among the other solutions, the need for the implementation of innovative landfill management techniques has increased the interest in bioreactor landfills as a viable in-situ treatment tool (Cossu, 2012; Reinhart et al., 2002).

A bioreactor landfill is typically defined as a system purposely planned and operated for the in-situ treatment 
of degradable waste with the aim of enhancing conversion processes. The possible in-situ measures include injection of air and/or water, leachate recirculation, and other combinations of in-situ treatments. These treatments create a more suitable environment for degradation processes by controlling biochemical kinetics, nitrification, moisture content, $\mathrm{pH}$, redox conditions, and gas emissions.

Moisture control particularly supports the metabolic processes, nutrients transport, microorganisms movement, and dilutes high concentration of inhibitors, while air injection speeds up the biodegradation processes and allows for the removal of nitrogen compounds (Cossu et al., 2003; Ritzkowski and Stegmann, 2013).

Bioreactor landfills can have several advantages over conventional landfills, from both an economic and environmental point of view:

- Reduce environmental impacts, by improving leachate quality and controlling landfill gas (LFG) emissions;

- The aftercare time is generally shorter due to the increased stabilisation rates therefore reducing aftercare costs and returning the site for different uses in a shorter timeframe;

- The leachate treatment is cheaper, since the in-situ treatment enhances leachate quality;

- The landfill gas (LFG) generation in an anaerobic bioreactor is enhanced;

- Refuse settlement and density are increased while less post-closure care operations are necessary (Berge et al., 2005; Omar and Rohani, 2015; Price et al., 2003; Warith, 2002).

On the other hand, a bioreactor landfill can have some disadvantages such as increased odours, physical instability of the waste mass due to the increase in moisture. Moreover, the need for aeration and/or leachate recirculation may increase capital and management costs.

According to the process, landfill bioreactors can be divided into four main types: anaerobic, aerobic, semi aerobic and hybrid. The hybrid bioreactor is a sequence of aerobic and anaerobic conditions (EPA, 2018a; Omar and Rohani, 2015).

Landfill bioreactors were mostly operated under anaerobic conditions (Price et al., 2003; Valencia et al., 2011; Vigneron et al., 2007) improving the methane generation rate, leachate quality, and reducing the period needed for long term maintenance and monitoring through recirculation, compared to traditional anaerobic landfills (Christensen, 2011). However, ammonia accumulation in leachate and the landfill body still remains one of the main challenges in anaerobic bioreactors. Furthermore, the anaerobic degradation process is still very slow.

According to the sustainable landfilling concept, the aerobic process is considered to be a better alternative to the traditional anaerobic landfills (Nikolaou et al., 2009; Read et al., 2001). Nevertheless, aerobic landfills are not always technically and economically feasible due to the need for forced ventilation systems, complex operation and management, and large energy consumption which translates to high operating and capital costs (Slezak et al., 2015). In order to overcome the cost disadvantage of forced aerated systems, the semi-aerobic landfill could be considered as an alternative solution to the aerated system (forced aeration). The semi-aerobic landfill aims to achieve aerobisation of the waste mass with a proper engineering design in which the ambient air naturally flows into the waste mass through leachate collection pipes, moved by the temperature gradient between the inside and outside of the landfill (Hanashima et al., 1981; Theng et al., 2005). Although developed at the Fukuoka University more than 20 years ago, this method is not widely spread around the world but field tested in Japan and in different on-going pilot projects in Italy, Pakistan, Iran, Nepal, Thailand, Malaysia, China, Vietnam, Samoa, and Mexico (Ministry of the Environment (Japan), 2018; JICA, 2004).

A limiting factor of aerobic bioreactors is the potential for complete inhibition of methane generation leading to the absence of any energy recovery. More recent developments have been shown in hybrid bioreactors, which are operated under various combinations of aerobic and anaerobic conditions (He et al., 2011; Long et al., 2009b; Sun et al., 2014; Xu et al., 2014). In a hybrid system, aerobic and anaerobic conditions can be purposely alternated to enhance the methane production for energy recovery and to achieve relatively faster waste stabilisation, facilitate conditions for nitrification and denitrification, improve leachate quality, reduce treatment costs (Berge et al., 2009), and potentially fulfil sustainability requirements. Bioreactor landfills are in some cases more economically advantageous than a traditional landfill (Berge et al., 2009; Hater et al., 2001; Theng et al., 2005), when accounting for landfill space recovery and a reduction in the post-closure care period (Anex et al., 1996).

A bioreactor landfill can also be operated as a flushing bioreactor. In a flushing bioreactor a large volume of water is applied in order to wash-out soluble waste constituents and accelerate waste stabilisation processes (Christensen et al., 2011). The magnitude of the flushing process is defined by the liquid to solid (L/S) ratio and according to Walker et al. (1997) the passage of approximately 4.6 times the bed volume of fluid is required to reduce leachate concentrations by two orders of magnitude, corresponding to a L/S ratio of $\sim 3 \mathrm{~m}^{3} / \mathrm{t}$ (Hupe et al., 2003; Christensen, 2011). However, the flushing process is strongly influenced by the solubility of various compounds in leachate (ammonia $\left(\mathrm{NH}_{4}\right)$, chemical oxygen demand (COD), Na, and Cl) (Christensen et al., 2011). Overall costs for this type of bioreactor may be two to four times higher than a conventional landfill (Karnik and Perry, 1997; Reinhart et al., 2002). Moreover the hydrodynamics of a landfill limits in time the potentialities of the flushing process. The high-water quantity addition increases the density of the waste, the hydraulic conductivity decreases and the short-circuiting phenomena tends to dominate with a limited portion of bulky waste subjected to water flow (Karnik and Parry, 1997; Walker et al., 1997).

The choice of the bioreactor landfill type is driven by the specific treatment objective to be achieved (e.g., energy recovery from landfill gas and/or leachate quality improvement) as well as by specific site conditions, such as waste 
characteristics, climate, and the social/economic situation. However, the sustainable landfill concept should be the driving principle in the bioreactor landfill design in order to assure the capability of achieving faster waste stabilisation (Cossu, 2010).

Several bioreactor landfill types have been successfully applied with promising results at lab or pilot scale, but full scale bioreactor landfills are still uncommon. The reasons for the lack of full scale systems are on one hand the regulatory constrains and on the other the technical complexity and cost investment associated with poorly demonstrated processes (Reinhart et al., 2002). This paper aims to review the state of the art bioreactor landfill research and elaborating on data to quantify the different kinetics with the goal of increasing the knowledge of bioreactors performances and potentialities.

Several literature lab-scale applications of different bioreactors have been analysed, compared, and an overview of different types is provided. The paper proposes a possible classification of the bioreactors, grouping them according to the main bioreactor types in literature, in order to simplify the bioreactors discussion. Advantages and disadvantages are discussed for each bioreactor category, although specific bioreactor performance should be considered individually. A qualitative analysis is then provided that takes into account some selected characteristics that are useful for the deciding on a specific bioreactor type such as methane production and energy recovery, biochemical kinetics velocity, nitrogen removal, technological complexity, and maintenance and leachate treatment costs. The ability for a bioreactor to achieve waste stabilization was quantified by the authors by mean of first-order kinetics which was determined by the approximation of the overall removal process of the selected relevant contaminants.

\section{DATA COLLECTION AND ELABORATION METHODOLOGY}

To provide an overall qualitative analysis of the different bioreactors types lab-, pilot- and full-scale applications of landfill bioreactors were considered. In order to quantify the stabilization performance and sustainability of the different systems, further and much more specific elaboration has performed based on lab-scale applications. Results from these studies have been published since 2005.

Variation kinetics of organic and nitrogen concentrations in leachate have been selected as criteria for the evaluation of the bioreactor stabilization performance (Ritzkowski et al., 2006) through the approximation of the combination of all the different processes involved in the stabilization of the bioreactor (e.g., biodegradation, flushing, volatilisation, etc.) in order to determine the overall first-order kinetics. These first-order kinetics were used for representing the removal process of the considered contaminants.

First-order kinetics (Heimovaara et al., 2014) for COD and ammonia conversion processes was performed by extrapolating the concentration values from graphs through the use of dedicated Matlab code and calibrating the following first-order kinetic equation:

$\mathrm{C}_{\mathrm{t}}=\mathrm{C}_{\text {peak }} * \mathrm{e}^{-\mathrm{kt}}$

where:

$\mathrm{C}_{\mathrm{t}}=$ concentration of considered contaminant at time $\mathrm{t}$

[mg/L];

$\mathrm{C}_{\text {peak }}=$ peak concentration $[\mathrm{mg} / \mathrm{L}]$;

$\mathrm{k}=$ kinetic constant $\left[\mathrm{d}^{-1}\right]$;

$\mathrm{t}=$ time of process $[\mathrm{d}]$

This equation is a strong approximation for a complete landfill simulation test (Fellner et al., 2009; Morello et al., 2017), but is acceptable for a qualitative discussion of the results of the investigated lab-scale tests. The concentration are clearly influenced by the water addition, but information about L/S ratio or water input were not clearly expressed in most of the cited papers. Starting from the data collection of the gas composition of the different bioreactors types, data elaboration has been performed in order to provide a graphical representation of the typical quality of the gases generated under different process conditions.

\section{DISCUSSION}

The results obtained from the leachate and gas literature data elaboration are presented in Table 1 and Figure 1. Peak and final concentrations $\left(\mathrm{C}_{\text {peak }}\right.$ and $\left.\mathrm{C}_{\text {end }}\right)$ of the considered contaminants in leachate are summarized in Table 1 for each analysed case study including the Putrescible Organic Fraction (POF) content of the studied waste. The $\mathrm{C}_{\text {peak }}$ has been considered as the beginning of the contaminant removal process, while the fraction of time required to reach the $\mathrm{C}_{\text {peak }}$ has been defined as Lag phase and has been indicated as the fraction of the whole experiment. COD and ammonia first-order kinetics have been calibrated to represent the contaminants removal process. In case where ammonia removal processes were not present, the related kinetics were not calculated.

Figure 1 summarises the typical composition of landfill gases under anaerobic, semi-aerobic, and aerobic conditions.

According to the U.S. EPA (2018), the contribution of landfills to the total non- $\mathrm{CO}_{2}$ GHGs emissions will count for approximately $7 \%$ of the total GHGs emissions worldwide by 2030 . The quality improvement of landfill gas represents a current challenge to limit the impact of landfills on climate change. The GHGs from landfill consist of primarily $\mathrm{CO}_{2}$ and $\mathrm{CH}_{4}$, along with several other trace gaseous components, such as non-methane volatile organic compounds (NMVOCs), nitrous oxide $\left(\mathrm{N}_{2} \mathrm{O}\right)$, nitrogen oxides (NOx), and carbon monoxide (CO). But only $\mathrm{CH}_{4}$ is counted towards a landfill's contribution to the GHG emissions (IPPC, 2006), being the most significant among the other emissions. In this study, the improvement of landfill gas quality performed by the landfill bioreactors has been considered only in terms of $\mathrm{CH}_{4}$ reductions. Nitrous oxide emissions can become an issue when bioreactor landfills are implemented, since both leachate recirculation (Price et al., 2003; Vigneron et al., 2007; Watzinger et al., 2005) 


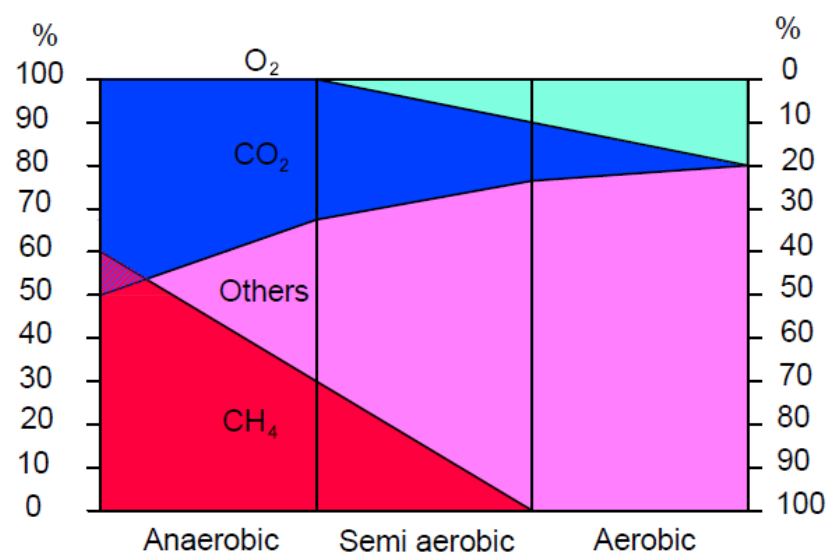

FIGURE 1: Composition of landfill gases in anaerobic, semi-aerobic and aerobic lab-scale bioreactors (Graph adapted using data from Ahmadifar et al. (2016), Borglin et al. (2004), Cossu et al. (2016, 2003), de Abreu et al. (2005); Erses et al. (2008), HUANG et al. (2008), Huo et al. (2008), Kim (2005), Nikolaou et al. (2008), Shao et al. (2008), Slezak et al. (2015), Sutthasil et al. (2014), Yang et al. (2012)).

and aeration (Berge et al., 2006; Powell et al., 2006; Tsujimoto et al., 1994) may induce $\mathrm{N}_{2} \mathrm{O}$ production. $\mathrm{N}_{2} \mathrm{O}$ production can result both from partial nitrification and partial denitrification (Mummey et al., 1994; Venterea and Rolston, 2000). Particularly, depending on the concentration of oxygen, the presence of oxygen during denitrification or oxygen below optimal levels during nitrification may result in the production of N2O (Berge et al., 2006; Khalil et al., 2004). A detailed study on the effects of the combination of the leachate recirculation and landfill aeration has been carry out by $\mathrm{He}$ et al. (2011). This study demonstrated the occurrence of $\mathrm{N}_{2} \mathrm{O}$ under different leachate recirculation and aeration conditions. However, results showed that the conversion of the total nitrogen added to columns into $\mathrm{N}_{2} \mathrm{O}$ occurred at a maximum of $0.18 \%$ and the significant reduction in nitrogen mass was mainly due to the production of $\mathrm{N}_{2}$. Moreover, although some $\mathrm{N}_{2} \mathrm{O}$ has been detected in several lab scale tests, the complete reduction of $\mathrm{N}_{2} \mathrm{O}$ to $\mathrm{N}_{2}$ can be expected within a full-scale landfill, due to the longer retention time of the gas (Price et al., 2003). Landfill $\mathrm{N} 2 \mathrm{O}$ is considered globally negligible, although these emissions may need to be considered locally in case of aerobic/ semi-aerobic bioreactor landfill.

\subsection{Anaerobic bioreactor landfills}

The anaerobic landfill bioreactor is the most common application of bioreactor systems where the biological degradation is enhanced by means of leachate recirculation and has been applied since the 80 s at several landfills in USA (Reinhart et al., 2002). The literature review of several lab-scale tests identified the peculiarities which are typical in all anaerobic bioreactors, regardless of the differences in the putrescible waste content. In particular the maximization of carbon removal occurs when methanogenesis starts. Once methane gas production increases, the concentrations of COD, five-day biochemical oxygen demand $\left(\mathrm{BOD}_{5}\right)$, and volatile fatty acids (VFAs) decrease and a subsequent rise in $\mathrm{pH}$ to the ranges of $6.8-8$ is observed.
The $\mathrm{BOD}_{5} / \mathrm{COD}$ ratio decreases from 0.8-0.4 to 0.4-0.1. The typical gas composition during the methanogenic phase shows between $30-60 \% \mathrm{CH}_{4}$ and $30-50 \% \mathrm{CO}_{2}$ (v/v) (Figure $1)$. These values are consistent with interstitial gas concentration in full-scale landfill bioreactors during the stable methanogenic phase (Raga and Cossu, 2014; Ritzkowski and Stegmann, 2007).

The main benefits associated with anaerobic bioreactors are both the increase in methane generation and the improvement of leachate quality compared to traditional landfills (Filipkowska, 2008; Read et al., 2001; Sanphoti et al., 2006). Sanphoti et al. (2006) compared the cumulative methane generation in anaerobic bioreactor with a traditional landfill. Anaerobic bioreactors with and without water addition generated $17 \mathrm{~L}_{\mathrm{CH} 4} / \mathrm{kg}_{\mathrm{TS}}$ and $54.9 \mathrm{~L}_{\mathrm{CH} 4} / \mathrm{kg}_{\mathrm{TS}}$ respectively, while only $9 \mathrm{~L}_{\mathrm{CH} 4} / \mathrm{kg}_{\mathrm{TS}}$ was produced in a traditional landfill simulation.

Despite the proven advantages associated with the anaerobic bioreactor compared to the traditional landfill, anaerobic bioreactors represent the least preferable option compared to the other bioreactor types when considering the concept of sustainability. The slow anaerobic degradation is confirmed by the lower COD and ammonia removal kinetics compared to other bioreactors (Table 1) which leads to contaminant emissions lasting for several decades in case of landfill gas and even for centuries in case of leachate (Rich et al., 2008; Ritzkowski et al., 2006). In particular the treatment of nitrogen in leachate remains to be the major challenge in aftercare, which is limitedly removed by flushing processes. Moreover leachate recirculation can even enhance ammonification, resulting in an increased ammonia concentration compared to traditional landfills (Berge et al., 2006; Long et al., 2009a; Price et al., 2003). This increase often causes the partial or complete inhibition of methane production, increases the costs for leachate treatment, and may create a significant long-term impact (Cossu et al., 2016).

Slow degradation rates and ammonia persistence puts the anaerobic bioreactor far from meeting sustainability requirements, threatens the public health and the environment over the long term and increases the costs associated with aftercare (Berge et al., 2006; Giannis et al., 2008; Read et al., 2001). Moreover, considering that a robust gas collection system is required in order to achieve a high collection efficiency, this infrastructure is not always technically and economically feasible in particular in developing countries (Sutthasil et al., 2014).

\subsection{Aerated bioreactor landfills}

Bioreactor landfills can be treated aerobically by injecting air in order to create an aerobic environment within the waste mass and to promote the growth of aerobic microorganisms. According to Ritzkowski and Stegmann (2012), different technologies and strategies have been developed for in-situ aeration, such as high pressure aeration, low pressure aeration, and active aeration with or without offgas extraction.

One of the first experiments on aerobic stabilization of municipal solid waste (MSW) was carried out by Stessel and Murphy (1992) to define the optimum air injection and 


\begin{tabular}{|c|c|c|c|c|c|c|c|c|c|c|c|c|c|c|c|c|c|c|c|c|c|c|c|c|c|}
\hline & 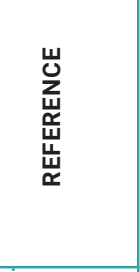 & $\mid$ & 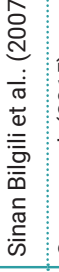 & 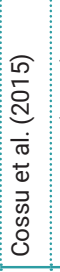 & 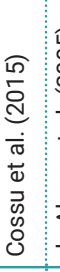 & 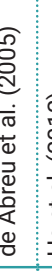 & 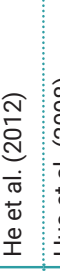 & 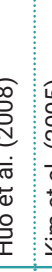 & 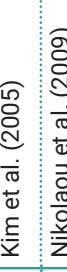 & 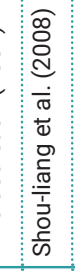 & 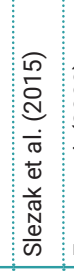 & 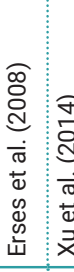 & 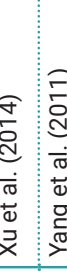 & & & 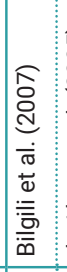 & 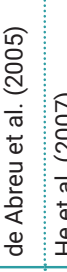 & 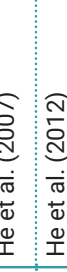 & 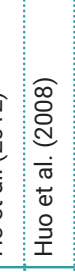 & 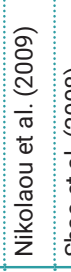 & 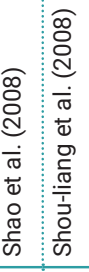 & 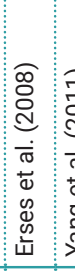 & 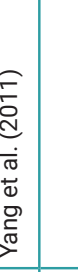 & & \\
\hline \multirow{5}{*}{$\begin{array}{l}\text { 号 } \\
\text { 商 } \\
\text { 至 }\end{array}$} & 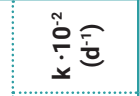 & & & 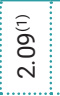 & 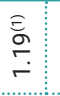 & 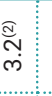 & & & & 离 & & & 产 & $\bar{N}$ & $\begin{array}{l}\infty \\
\infty \\
0 \\
0\end{array}$ & & 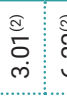 & సู๊ & & & 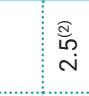 & & 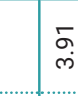 & õ & \\
\hline & 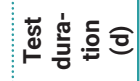 & & & $\hat{\mathrm{m}}$ & ô & $\frac{\circ}{m}$ & & & & $\stackrel{\sim}{\sim}$ & & & ৪্ & & & & $\stackrel{\circ}{m}$ & 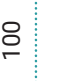 & & & $\stackrel{\sim}{\sim}$ & & & & \\
\hline & تृ & & & $\simeq$ & $m$ & $\infty$ & & & & $\stackrel{\infty}{\sim}$ & & & n & & & & $\infty$ : & $\bar{m}$ & & & $\stackrel{\infty}{\sim}$ & & & & \\
\hline & 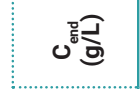 & & & 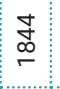 & 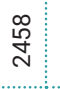 & is & & & & 各 & & & 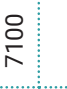 & & & & $\dot{N}$ & શิ & & & $\stackrel{ }{\sim}$ & & & & \\
\hline & 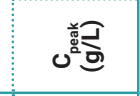 & & & 察 & $\begin{array}{l}8 \\
\text { o } \\
\circ\end{array}$ & ָָ & & & & i & & & ষ্ণ & & & & d্ & g & & & 옹 & & & & \\
\hline \multirow{5}{*}{ 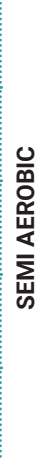 } & 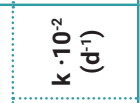 & 袋 & & & & & 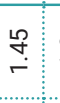 & $\stackrel{\leftrightarrow}{r}$ & & & & & 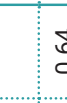 & $\begin{array}{c}\text { ț } \\
0\end{array}$ & 菅 & & & 8 & $\stackrel{\infty}{\infty}$ & & 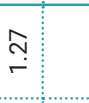 & & \begin{tabular}{c|c}
$\infty$ & $\stackrel{\infty}{\infty}$ \\
0 & $\stackrel{r}{-}$
\end{tabular} & 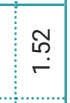 & \\
\hline & 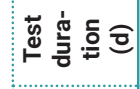 & $\stackrel{ }{ }$ & & & & & న & 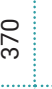 & & & & & s & : & & & & $\frac{8}{2}$ & $\hat{~}$ & & ৪ి & & ঃু & & \\
\hline & す马ّ & 0 & & & & & a & 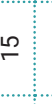 & & & & & + & & & & & 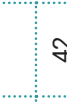 & $y$ & & ৪ి & & $\sim$ & & \\
\hline & 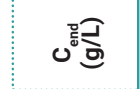 & : & & & & & : & ลิ & & & & & 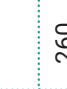 & : & & & & 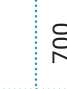 & R & & N & & in & & \\
\hline & 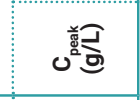 & 咅 & & & & & $\begin{array}{ll}8 \\
\\
0\end{array}$ & 㤂 & & & & & $\begin{array}{l}1 \\
0 \\
0\end{array}$ & & & & & $\frac{1}{2}$ & $\frac{\infty}{\infty}$ & & o & & 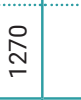 & & \\
\hline \multirow{5}{*}{$\frac{0}{0}$} & 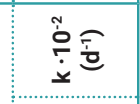 & $\stackrel{\operatorname{Ln}}{+}$ & 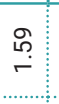 & & & & & & 赵 & & $\stackrel{\stackrel{m}{N}}{i}$ & $\underset{\substack{\infty \\
\leftarrow}}{+}$ & & is & $\frac{m}{\dot{f}}$ & : & & & & $\stackrel{N}{m}$ & ر? & $\stackrel{\text { f }}{-}$ & $\stackrel{\bar{m}}{i}$ & o. & \\
\hline & 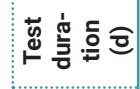 & $\stackrel{\circ}{\circ}$ & 品 & & & & & & 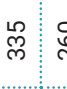 & & $\stackrel{\circ}{\circ}$ & $\stackrel{\infty}{\infty}$ & & & & 品 & & & & 8 & ฉి & $\stackrel{\infty}{m}$ & & & \\
\hline & すす & $m$ & 0 & & & & & & $\stackrel{\circ}{\circ}$ & & is & $\diamond$ & & & & $\stackrel{i}{\sim}$ & & & & a & 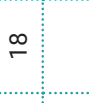 & $\circ$ & & & \\
\hline & 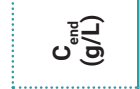 & 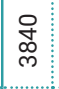 & : & & & & & & 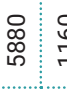 & & : & t & & & & స్ి & & & & $N$ & প্ল & is & & & \\
\hline & 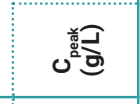 & 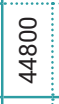 & $\begin{array}{l}\text { 总 } \\
\text { 品 } \\
\text { o }\end{array}$ & & & & & & ঃ & & 布 & 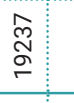 & & & & 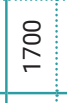 & & & & 암 & g & $\hat{q}$ & & & 1 \\
\hline & 苞 & 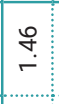 & $\begin{array}{c}\infty \\
\circ \\
0 \\
0\end{array}$ & $\stackrel{\circ}{\stackrel{\circ}{\leftarrow}}$ & & $\stackrel{\stackrel{9}{:}}{\stackrel{2}{r}}$ & 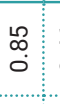 & $\begin{array}{l}0 \\
0 \\
0\end{array}$ & $\overline{0} ?$ & 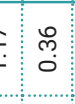 & 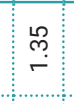 & $\stackrel{n}{0}: \frac{1}{c}$ & $\stackrel{1}{\circ}$ & 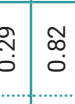 & $\begin{array}{l}\stackrel{0}{0} \\
0 \\
0\end{array}$ & Ni & ô & 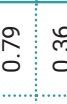 & $\begin{array}{l}0 \\
:\end{array}$ & o & $\stackrel{\infty}{\circ}$ & $\frac{t}{0}$ & 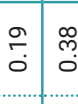 & $\stackrel{\infty}{\sim}$ & 吾 \\
\hline & $\stackrel{\square}{*}$ & $\stackrel{\circ}{\circ}$ & in & مि & 吕 & $\stackrel{\circ}{m}$ & ה & 尽! & 웡 & 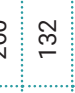 & $\stackrel{\stackrel{\leftrightarrow}{\circ}}{\circ}$ & ᄋ్ర ণ & ৪্ল & : & & : & $\stackrel{\circ}{m}$ & 8 & is & $\stackrel{O}{N}$ & ণ্ল & পి & ৪ু & & 종 \\
\hline & ฮั & $m$ & $\stackrel{m}{\square}$ & 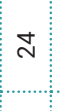 & & م & 의 & $\stackrel{\circ}{\circ}$ & $+\circ$ & in & $\stackrel{m}{\square}$ & $\circ$ & in & s & & के & $\infty$ & 6 & y & $\simeq$ & $\stackrel{\infty}{\circ}$ & รั & $\sigma$ & & 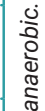 \\
\hline & ن & 点 & : & 各 & & : & 吕 & 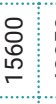 & 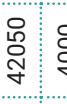 & : & : & ০ & 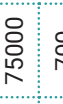 & 8 & & 염 & 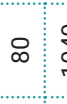 & \begin{tabular}{l} 
f: \\
0 \\
\hdashline
\end{tabular} & : & 另 & 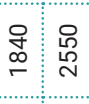 & $\infty$ & $\frac{0}{i n}$ & & 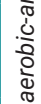 \\
\hline & U. & $\begin{array}{l}\text { Oे } \\
\text { ôे }\end{array}$ & o: & 葴 & & รู & 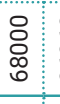 & 齐: & 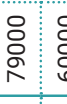 & : & $\begin{array}{l}\text { o } \\
\text { o } \\
\end{array}$ & 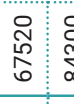 & $\begin{array}{lll}8 & 0 \\
o \\
\infty \\
\infty\end{array}$ & 8 & & $\stackrel{\circ}{\circ}$ & 虽 & 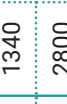 & : & 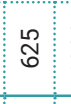 & ঃু & $\stackrel{+}{\circ}$ & $\begin{array}{c}\stackrel{8}{\infty} \\
\stackrel{\rho}{\circ}\end{array}$ & & 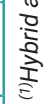 \\
\hline \multicolumn{2}{|r|}{ แ艹 } & $\infty$ & $\forall$ & $\triangleright$ & & $\triangleright$ & $\stackrel{\sim}{\sim}$ & 尺 & $\stackrel{\circ}{\circ}$ & $\circ$ & $\stackrel{\infty}{\infty}$ & 过 & 号 c & 苂 & & $\forall$ & $\stackrel{\Delta}{t}$ & g) & $R$ & 8 & $8 \circ$ & \& & 帝 & & $\frac{\text { di }}{9}$ \\
\hline & 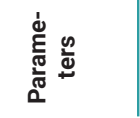 & & & & & & & & & & & & & & & & & & & & & & & 这的 & \\
\hline
\end{tabular}


leachate recirculation rate for degradation. Faster stabilization and improved settlement were demonstrated (Stessel and Murphy, 1992).

The positive effects of aeration on waste stabilization have been confirmed by several studies by comparing anaerobic and aerobic conditions (Table 1). Despite variation in the POF, lab-tests revealed similar results in terms of stabilization performance with higher carbon and nitrogen removal kinetics and/or shorter lag phase compared to anaerobic conditions. Aeration also lowered the leachate carbon and nitrogen values and achieved a final BOD $_{5} / C O D$ ratio between 0.02-0.003 (Table 1 ). Volatile organic acids production decreased by limiting the anaerobic fermentation processes and resulted in $\mathrm{pH}$ ranges between 6-8 after the initial acidic phase.

Although the aerobisation (establishment of aerobic conditions) of the waste mass prevents methane generation and thus energy recovery, there are several advantages compared to the anaerobic bioreactor landfill, which can be summarised as follows:

- Acceleration of the degradation processes in the landfill due to the higher biochemical aerobic degradation kinetics, reducing the long-term emission potential as well as the post closure management costs. In addition to faster settlement of the landfill, the site can be used for other uses in shorter time period (Yuen et al., 1999, Read, 2001);

- Higher waste settlement that generates additional landfill capacity

- Reduction of leachate volumes and enhanced remediation of recalcitrant carbon molecules and nitrogen compounds, improving the leachate quality resulting in the subsequent financial savings for secondary treatment;

- Reduction of $\mathrm{CH}_{4}$ generation and increased carbon gasification dominated by $\mathrm{CO}_{2}$;

- Reduction of odours generally produced from anaerobic degradation, such as hydrogen sulphide and volatile acids (Jacobs et al., 2003).

Among others, nitrogen removal is one of the most significant benefit of an aerobic system. In anaerobic landfills, nitrogen removal from leachate, in form of ammonia ion, is generally performed ex situ using costly and complex treatment plants. In order to avoid these costs, in-situ techniques have become an attractive solution and to date the most used alternative is the aeration of the waste mass to facilitate nitrification-denitrification processes (Berge et al., 2006; Shao et al. 2008). Although air injection will theoretically inhibit the denitrification process, the complete aerobisation of the waste mass is never achieved in the field. Therefore anaerobic and anoxic areas still exist inside the landfill and both processes can take place simultaneously even under low biodegradable matter conditions (Berge et al., 2006; Giannis et al., 2008; Ritzkowski, 2011; Ritzkowski and Stegmann, 2005, 2003; Shao et al., 2008). Air stripping and volatilisation can also occur since these processes are favoured by higher $\mathrm{pH}$ levels and temperatures reached in an aerobic system and can also be facilitated through the gas flow associated with air injection (Berge et al., 2005).
The forced air flow and the temperature rising up to more than $60^{\circ} \mathrm{C}$ results in a high evaporation of water and in a low quantity of leachate (Berge et al., 2005; Read et al., 2001).

Recirculation still represents an additional in situ leachate treatment tool to improve stabilization performance (Sinan Bilgili et al., 2007). In particular, the increased frequency of leachate recirculation accelerates the stabilization rate of waste, even if too much recirculation leads to saturation, ponding, and acidic conditions (Šan and Onay, 2001). Slezak et al. (2015) observed that the higher recirculation rate, increased the reduction of carbon and nitrogen parameters in leachate over a shorter time period but $\mathrm{O}_{2}$ diffusion was limited leading to lower waste stabilization.

Aeration rates and modes influence the degradation performance differently. Slezak et al. (2010) compared stabilization performance of four aerobic lysimeters with different aeration rates obtaining similar changes in leachate parameters and demonstrated that above the minimum aeration requirements the increased rates do not provide any additional benefits. Intermittent aeration has been demonstrated to be much more effective than continuous aeration (Cossu et al., 2016; Morello et al., 2017); however optimum aeration rate is strongly influenced by oxygen consumption, which varies according to waste composition, age, and operating parameters.

Fate of metals in aerobic and anaerobic landfill bioreactors was investigated by Kim et al. (2011). Apart from the initial acidic phase, heavy metals mobility was reduced under aerobic conditions due to the high $\mathrm{pH}$ and positive redox conditions, affecting solubility and sorption properties. Metals were retained in the waste by sorption, carbonate precipitation, and hydroxide precipitation (Borglin et al., 2004; Giannis et al., 2008).

Typical composition of off gases reported in lab scale tests consists of $10-20 \% \mathrm{O}_{2}$ and $0-20 \% \mathrm{CO}_{2}$ (Figure 1). Methane generation is almost completely inhibited under aerobic conditions and mostly $\mathrm{CO}_{2}$ is produced (Mertoglu et al., 2006; Slezak et al., 2015). On one hand aerobic conditions impede energy recovery while on the other environmental impacts are limited when biogas collection and control is not technically or economically feasible and uncontrolled emissions are expected. Ritzkowski and Stegmann (2007) demonstrated that in situ aeration could avoid more than $72 \%$ of the total $\mathrm{GHG}$ emissions occurring under anaerobic conditions.

Since the faster waste stabilization under aerobic conditions, carbon gasification is enhanced. Slezak et al. (2015) compared $\mathrm{CO}_{2}$ and $\mathrm{CH}_{4}$ gasification from anaerobic and aerobic lysimeters. The results showed that carbon gas released from aerobic lysimeters was about 5 times higher than that the one from anaerobic ones.

Potential disadvantages, which limit the use of this technology are the risks associated with the drying of the waste mass due to the high temperatures which may limit the highly sensitive nitrogen removal biological processes and may create an elevated temperature or fire potential. However, limited methane production, proper moisture content, and waste pre-treatment can overcome these problems (Berge et al., 2005). The high costs due to the 
energy requirements for compressed air injection may be limited by the appropriate selection of operating parameters, including aeration and recirculation rates, providing optimum conditions for waste decomposition, and minimizing energy consumption (Rich et al., 2008). According to the hypothetical cost model developed by Read et al. (2001), aerobic landfills could be a cost-effective solution when considering the potential recovery of valuable materials from the site, even if the operational costs and the regulatory requirements of closed landfills represents an obstacle for the full-scale development of aerobic landfills (Read et al. 2001).

Forced aeration is nowadays mostly used for remediating old anaerobic landfill, instead of being only a designed option for active landfill management. This is because aeration of old landfills represents a feasible solution to biologically stabilize waste, reduce nitrogen concentrations, and significantly control liquid emissions (Hrad et al., 2013; Ritzkowski and Stegmann, 2005, 2003). Moreover, the aeration of the landfill mass is a fundamental pre-treatment for landfill mining procedures (Raga and Cossu, 2014; Ritzkowski and Stegmann, 2012). In remediation, this technology is generally preferred over flushing: although on one hand flushing has been demonstrated to be the most effective approach (Bolyard and Reinhart, 2015), on the other hand it requires large volumes of water, off-site leachate treatment costs, and is not always technically or economically feasible (Ritzkowski et al., 2006).

Combination of both flushing and aeration processes however, have been suggested as alternative landfill management approaches by Cossu et al. (2003). PAF model was proposed as a combination of mechanical-biological Pre-treatment with Aeration and Flushing to exploit the advantages of the individual options. PAF and flushing reactors were compared to the traditional anaerobic, semi aerobic, and aerated landfills. Among the others, flushing bioreactors revealed faster kinetics and lower concentration values for carbon and nitrogen control parameters, even if the aerobic reactor presented lower residual carbon in the final solids and greater gasification. Gas generation is limited in flushing reactors since the washing of waste tends to remove the soluble biodegradable substance available to gasification (Cossu et al., 2003; Purcell et al., 1997).

\subsection{Semi aerobic bioreactor landfills}

The semi-aerobic system has been developed in Japan by Hanashima (1961). This system could be considered as a lower cost alternative solution to the aerobic landfill system, by providing the same benefits but lowering the operational costs by avoiding the direct air injection. Aerobic bacteria activity is improved by the natural flow of the external air into the waste mass through the leachate collection pipes, moved by the temperature gradient between the inside and outside of the landfill (Theng et al., 2005). The movement of air is particularly enhanced in winter and during the night when the temperature differences are higher. Hirata et al. (2012) observed that aerobic bacteria count in semi-aerobic systems were higher compared to anaerobic bacteria, demonstrating the effectiveness of the semi-aerobic system in the aerobisation of the waste mass.
Reproducing the aerobic process, the semi-aerobic system achieves the same benefits described for the aerated bioreactor landfill which has been proved by several labscale studies as well as by large-scale applications.

According to the data elaboration presented in Table 1, results show that regardless of the differences in the POF fraction of waste, the semi-aerobic system is able to achieve a much higher organic matter stabilization than the anaerobic system. The COD and ammonia concentrations in the leachate are always lower under semi-aerobic conditions, achieving higher removal kinetics. In particular, ammonia oxidation was achieved by creating aerobic conditions, while the simultaneous presence of anaerobic, anoxic, and aerobic zones within the waste mass creates conditions for denitrification of the nitrate. Shao et al. (2008) obtained higher efficiency under semi-aerobic conditions rather than in fully aerobic bioreactor since denitrification was limited due to the persistent presence of oxygen.

Despite the capability of the semi-aerobic system to partially simulate aerobic conditions, aerated bioreactors remain the best performing systems in terms of COD concentrations, degradation rates, and removal efficiencies (Table 1) (Ahmadifar et al., 2015).

$A$ benefit of the aerobisation of the waste mass is the higher gasification occurring under semi-aerobic conditions dominated by $\mathrm{CO}_{2}$ (Figure 1). According to Matsufuji et al. (1996) the proportion of gas to leaching emissions was 3:2 from the semi-aerobic lysimeter and 1:4 from anaerobic lysimeters. Similar results were obtained by Shimaoka et al. (2000) with a ratio of $4: 1$ and 2:3 under semi-aerobic and anaerobic conditions, respectively. Lavagnolo et al. (2018) achieved up to a $60 \%$ initial carbon gasification under semi-aerobic conditions compared to only $20 \%$ in anaerobic reactors.

Intergovernmental Panel on Climate Change (IPCC) guidelines (IPCC, 2006) estimates that the degradation process within a semi aerobic waste mass is supposed to occur simultaneously under anaerobic and aerobic conditions in line with the heterogeneity of the waste mass. According to this, the biogas composition in a semi-aerobic landfill is described by a $\mathrm{CH}_{4} / \mathrm{CO}_{2}$ ratio of 0.48 (Jeong et al., 2015). This value seems to align well with the majority of the values reported in the literature. The average methane concentration in the semi-aerobic process mostly ranges between $0-30 \%(\mathrm{v} / \mathrm{v})$ with $\mathrm{CO}_{2}$ and $\mathrm{O}_{2}$ at $10-30 \%(\mathrm{v} / \mathrm{v})$ and 0-20\% (v/v), respectively (Figure 1 ).

\subsection{Hybrid bioreactors}

Hybrid bioreactors are conceptually based on the principle of combining a sequence of aerobic and anaerobic conditions with the purpose of achieving the benefits from both conditions in order to maximise the potential of bioreactors in terms of sustainability and/or methane generation. In particular methane production and energy recovery are maximized during the anaerobic phase while during the aerobic phase the nitrification-denitrification processes are enhanced for complete removal of nitrogen from landfill. Overall waste stabilization is achieved in a shorter period of time by improving the degradation of recalcitrant compounds such as lignin and aromatic substances 
(Berge et al., 2006, 2005; He et al., 2011; Long et al., 2009b; Ritzkowski and Stegmann, 2013; Sun et al., 2013). A challenge with a Hybrid Bioreactor is the economic cost since continuous injection-extraction plants are expensive or alternatively require a biological leachate treatment plant. Consequently, this technology is applied for limited periods of time when traditional degradation processes cannot decrease the pollution any further (Berge et al., 2006). However, the high maintenance costs associated with air injection and leachate recirculation are generally covered by the increasing methane generation and/or by leachate treatments savings due to recirculation and aeration (Berge et al., 2009). Several different hybrid conditions have been tested at lab scale with promising results through combining various sequences of aerated and non-aerated phases, aeration modes (continuous or intermittent), and application (leachate aeration or in situ waste aeration).

\subsubsection{Anaerobic-Aerobic sequencing}

Long et al. (2009) proposed a hybrid bioreactor landfill sequencing the anaerobic and aerobic phases. At the end of the second phase, the system was able to achieve more than a $97 \%$ removal efficiency of COD and ammonia, nitrifying and denitrifying more than $70 \%$ of the initial content of nitrogen in the waste sample, produced methane for energy recovery, and dropped the main pollutants concentration to low levels (COD < $400 \mathrm{mg} / \mathrm{L}$ and ammonia < $20 \mathrm{mg} / \mathrm{L})$. Aerobic conditions through air injection significantly improved the stabilization of the refuse, the readily biodegradable organic matter was mineralized during the initial anaerobic phase, and the hardly biodegradable organic matter was stabilized mainly during the aerobic phase. Ammonia was converted to $\mathrm{NO}_{3}^{-}$and $\mathrm{NO}_{2}^{-}$in ex-situ nitrification, while nitrate was reduced into nitrite and then to $\mathrm{N}_{2}$ gas in in-situ denitrification. A simple example of the application of the hybrid bioreactor is the aeration of old landfills, in which the long lasting anaerobic process occurred over the lifetime of the landfill is followed by forced aeration. Forced aeration is an efficient technology applied worldwide for the remediation of persistent pollution (Ritzkowski and Stegmann, 2013). The same has been applied in some more recent landfills which were built as anaerobic bioreactors in order to achieve methane production leaving the possibility of applying in-situ aeration as a subsequent phase. This type of operation would convert this landfill to a Hybrid Bioreactor.

\subsubsection{Aerobic-Anaerobic}

When aerobic-anaerobic sequencing is applied completely in situ, aeration could be addressed to maximize the methane production by accelerating the initial acidogenic phase and anticipating optimum $\mathrm{pH}$ and VFA conditions for methanogenesis (Xu et al., 2014; Morello et al., 2017). Mali Sandip et al. (2012) showed that pre-aeration in combination with leachate recirculation and/or inoculum injection could increase the methane production by $25 \%$. Similar results were obtained by Xu et al. (2014) using a lab scale hybrid bioreactor with intermitted air injection before a second anaerobic phase which achieved a higher methane production (about $32 \mathrm{~L}_{\mathrm{CH}_{4}} / \mathrm{kg}_{\mathrm{TS}}$ ) and a higher consumption of organic compounds compared with a full anaerobic one in which methane production never started due to excessive acidity. Aeration frequencies, depth and rates strongly influence the methane production, the decomposition of organic carbon, and nitrification. Xu et al. (2015) operated two hybrid bioreactors with two different initial aeration frequencies (twice and 4 times per day) with same unit rate of $0.1 \mathrm{~L} / \mathrm{min} / \mathrm{kg}_{\mathrm{TS}}$ until $\mathrm{pH}>7$, obtaining similar trends in COD and ammonia values but higher methane generation in the case of low frequency aeration (85 $\mathrm{L}_{\mathrm{CH} 4} /$ $\mathrm{kg}_{\mathrm{TS}}$ compared to $72 \mathrm{~L}_{\mathrm{CH} 4} / \mathrm{kg}_{\mathrm{TS}}$ ). Cossu et al. (2015) tested aerobic-anaerobic hybrid bioreactors with continuous and intermittent aeration until optimum $\mathrm{pH}$ and VFA concentrations for methanogenesis were achieved. Both aeration modes were beneficial in accelerating waste stabilization and the acidogenic phase, however intermittent aeration until optimum $\mathrm{pH}$ values was more efficient in enhancing stabilization kinetics and methane generation (Table 1). According to Wu et al. (2014), aeration at the bottom layer achieved enhanced decomposition of organic carbon, while high air injection rates lead to effective simultaneous nitrification-denitrification. This combination accelerated waste decomposition but may limit methane generation. Despite the cited benefits of pre-aeration, it does not solve the problem of persistent nitrogen pollution in leachate and in all previous studies strong ammonification occurs during the first aerobic phase with positive trend in ammonia concentration which accumulated during the second anaerobic phase (Cossu et al., 2016; He et al., 2011; Morello et al., 2017; Xu et al., 2015, 2014). For this reason, S.An.A landfill model has been suggested, including a third final phase of post-aeration to drop down nitrogen indexes in leachate (Cossu et al., 2016; Morello et al., 2017). The Semiaerobic-Anaerobic-Aerobic (S.An.A) Landfill model is a hybrid system with an initial semi-aerobic phase to enhance the methane production occurring in the anaerobic step which is then followed by forced aeration for the abatement of the residual emissions. According to Morello et al. (2017) with this approach it was possible to achieve a methane potential $50 \%$ higher than that of a traditional anaerobic bioreactor which equates to an estimated reduction of aftercare by $25-35 \%$.

A Mechanical Biological Pre-treatment (MBP) of waste before anaerobic landfilling could be regarded as a form of a hybrid bioreactor, with off-site forced aeration followed by in situ anaerobic reactions. MBP aims to achieve a quick stabilization of the waste and during landfilling the production of landfill gas might not be significant for energetic exploitation.

\subsubsection{Facultative landfill}

In order to overcome the challenge of ammonia accumulation under anaerobic conditions, an alternative solution consists of an external aerobic pre-treatment of leachate prior to recirculation in an anaerobic bioreactor, to allow for simultaneous nitrification and denitrification to occur in order to remove nitrogen compounds (Berge et al., 2005; de Abreu et al., 2005; Price et al., 2003; Zhong et al., 2009). This system aims at ensuring that the energy recovery due to methane production is maintained throughout 
the whole landfill by facilitating anaerobic conditions. In order to remediate nitrogen pollution in the leachate, the leachate is aerobically treated to nitrify the ammonia and then it is re-injected into the landfill to denitrify the produced nitrates. This system is also patented in the United States (US639895, 2002) by the name of a facultative landfill and has been tested at the lab scale by Price et al. (2003) in order to verify that the bioreactor is capable of denitrifying the nitrates produced during aerobic leachate treatment. The options available for ex situ leachate treatment are chemical-physical (ion-exchange, air stripping, chemical precipitation, reverse osmosis) and biological. Among the others, biological treatment is the most common since costs are limited compared to other processes (He et al., 2007). Several lab scale ex situ biological leachate nitrification options have been studied including the aerobic biofilter (Jokela et al., 2002), sequential anaerobic and air-lift loop sludge blanket reactors (He et al., 2007), continuous stirred tank reactor (Zhong et al., 2009), activated sludge reactor (Huo et al., 2008), fluidized bed reactors (de Abreu et al., 2005), and aerobic landfill reactor (Sun et al., 2017). All these studies demonstrate the capability of the facultative bioreactors to remove nitrogen through ex-situ nitrification of $\mathrm{NH}_{4}$ to $\mathrm{NO}_{2}$ and $\mathrm{NO}_{3}$ and in-situ denitrification to convert nitrates to $\mathrm{N}_{2}$ gas.

De Abreu et al. (2005) compared the performance of an anaerobic bioreactor with that of a facultative bioreactor with external aerobic biological leachate treatment consisting of an electrocoagulation/settling unit for metals removal and two fluidised bed reactors. According to Table 1 there are clear benefits in both COD and ammonia removal observed in the facultative bioreactor with higher removal kinetics (1.8-fold and 7.7-fold for the anaerobic column for COD and ammonia, respectively), achieving a final COD and $\mathrm{NH}_{4}$ concentration much lower compared to the anaerobic bioreactors. Shou-liang et al. (2008) compared the performances of an anaerobic bioreactor with those of a facultative bioreactor. The latter consisted of a fresh waste landfill reactor for denitrification, a well decomposed waste landfill reactor for methanogenesis, and an aerobic-activated sludge reactor for nitrification. The obtained results showed the capability of the system to improve the methane generation and promote ammonia removal since nitrification and subsequent denitrification occurred with removal kinetics 8-folds higher than anaerobic conditions. The acidogenic phase was accelerated in the hybrid reactor with a higher methane concentration during the experimental period, while inhibiting methanogenesis in the anaerobic reactor due to the VFA accumulation and low $\mathrm{pH}$ level. He et al. (2007) studied the performance of a facultative reactor with an external leachate treatment consisting of a sequential up flow anaerobic sludge blanket reactor for organic matter removal and an air-lift loop sludge blanket reactor for nitrification. Even if the COD removal was quite similar to the control reactor, the ammonia removal was strongly enhanced with final $\mathrm{NO}_{3}$ values of about $4 \mathrm{mg} / \mathrm{L}$, suggesting the occurrence of denitrification. This kind of Hybrid Bioreactor is promising because it allows for the reduction in ammonia in the landfill without any aeration systems while ensuring methane recovery at the same.
The downside of this process is the continued need for a biological leachate treatment plant.

The high concentration of nitrate produced in ex-situ nitrification may inhibit methanogenesis in a facultative bioreactor. For this reason, Sun et al. (2017) studied the use of ex situ simultaneous nitrification-denitrification in an aged refuse bioreactor for nitrification prior to in-situ denitrification, in order to enhance the methane production. Hirata et al. (2012) proposed the SeRA system (recirculatory semi-aerobic landfill) with ex situ leachate aeration in order to improve the semi-aerobic landfill performance by reducing the in situ oxygen demand, expanding the aerobic zone in the waste mass, and improving the nitrification denitrification process. SeRA achieved a similar TOC degradation performance compared to the aerobic lysimeter and an even better total nitrogen degradation performance confirmed by the higher gasification rates.

\section{BIOREACTORS COMPARISON IN TERMS OF SUSTAINABILITY}

A comparative qualitative analysis of bioreactor types are summarised in Table 2 based on selected characteristics, such as persistent emissions, technological complexity, maintenance costs, and leachate treatment costs.

Considering the prior need of achieving landfill sustainability, ammonia is generally recognized as the main long-term pollutant in leachate. Therefore almost all the bioreactor types involved some form of a nitrification-denitrification process with different methodologies. Even if the carbon and nitrogen emissions can be reduced efficiently, leachate can also be polluted by saline compounds and heavy metals, which are difficult to be removed biologically.

The performance of each type of bioreactor may highly depend on the-situ conditions, such as waste characteristics and climate, which should be taken into consideration beyond the objectives to be pursued (i.e. energy recovery, faster waste stabilization, washing of soluble compounds). For example, according to the recent European Regulations (EU, 2015), the reduction of the POF in landfilled waste and waste pre-treatment limit the practicability of bioreactors that are intended for energy recovery, while these bioreactors will surely have a central role in waste management outside of Europe (Reinhart et al., 2002). Moreover, the capability of bearing the costs and the technological complexity will strongly depend from country to country. Nevertheless, knowing the general behaviour in stabilization performance of each bioreactor type at the lab scale may help to identify the best bioreactor solution at field scale. The best performance would be based on the aim to fulfil the sustainability concepts according to the specific site objectives and in-situ conditions. For this reason, the quantification of the stabilization performance and thus the sustainability of the different systems has been carried out.

According to Berge et al. (2009), the main parameters that influence bioreactor economics are air space recovery, gas recovery for the subsequent energetic use, and savings resulting from reduced leachate treatment requirements. Therefore, faster biological stabilization provides a metric for measuring the successfulness of any landfill 
TABLE 2: Qualitative analysis of different landfill bioreactor types compared to the traditional landfill.

\begin{tabular}{|c|c|c|c|c|c|c|c|}
\hline \multirow[b]{2}{*}{$\begin{array}{l}\text { Bioreactor } \\
\text { Landfill Type }\end{array}$} & \multicolumn{2}{|c|}{ Objective } & \multirow[b]{2}{*}{$\begin{array}{l}\text { Biochemical } \\
\text { Kinetics }\end{array}$} & \multirow[b]{2}{*}{$\begin{array}{l}\text { Other Persistent } \\
\text { Emissions }\end{array}$} & \multirow[b]{2}{*}{$\begin{array}{l}\text { Technological } \\
\text { complexity }\end{array}$} & \multirow[b]{2}{*}{$\begin{array}{l}\text { Maintenance } \\
\text { Costs* }\end{array}$} & \multirow{2}{*}{$\begin{array}{l}\text { Leachate } \\
\text { treatment } \\
\text { costs * }\end{array}$} \\
\hline & $\begin{array}{c}\text { Methane } \\
\text { production \& } \\
\text { energy recovery }\end{array}$ & $\begin{array}{l}\text { Nitrogen } \\
\text { removal }\end{array}$ & & & & & \\
\hline $\begin{array}{l}\text { Traditional } \\
\text { Landfill }\end{array}$ & $\begin{array}{l}\text { Traditional } \\
\text { Recovery }\end{array}$ & by leaching & slow & $\begin{array}{l}\mathrm{NH}_{4}^{+} \text {, Salinity, } \\
\text { Heavy metals }\end{array}$ & Gas collection & Low & High \\
\hline Anaerobic & $\begin{array}{l}\text { Enhanced } \\
\text { recovery }\end{array}$ & by leaching & Medium-slow & $\begin{array}{l}\mathrm{NH}_{4}^{+} \text {, Salinity, } \\
\text { Heavy metals }\end{array}$ & $\begin{array}{l}\text { Leachate } \\
\text { recirculation, Gas } \\
\text { collection }\end{array}$ & $\begin{array}{l}\text { Leachate } \\
\text { recirculation }\end{array}$ & $\begin{array}{l}\text { Savings from } \\
\text { leachate } \\
\text { recirculation }\end{array}$ \\
\hline Aerobic & No & Nitro-Denitro & fast & $\begin{array}{l}\text { Salinity, Heavy } \\
\text { metals }\end{array}$ & $\begin{array}{l}\text { Leachate } \\
\text { recirculation, Air } \\
\text { Injection }\end{array}$ & $\begin{array}{l}\text { Air injection, } \\
\text { Leachate } \\
\text { recirculation }\end{array}$ & $\begin{array}{l}\text { Savings from } \\
\text { leachate } \\
\text { recirculation and } \\
\text { aeration }\end{array}$ \\
\hline Semi-aerobic & No & $\begin{array}{l}\text { Partial Nitro-de- } \\
\text { nitro }\end{array}$ & medium & $\begin{array}{l}\text { Salinity, Heavy } \\
\text { metals }\end{array}$ & $\begin{array}{c}\text { Build to enhance } \\
\text { natural } \\
\text { convection }\end{array}$ & $\begin{array}{l}\text { Sometimes } \\
\text { Leachate } \\
\text { recirculation }\end{array}$ & $\begin{array}{l}\text { Savings from } \\
\text { aeration }\end{array}$ \\
\hline Hybrid & $\begin{array}{l}\text { Enhanced } \\
\text { recovery }\end{array}$ & Nitro-Denitro & $\begin{array}{c}\text { fast } \\
\text { (limited for } \mathrm{NH}_{4} \\
\text { in aerated-anaer- } \\
\text { obic) }\end{array}$ & $\begin{array}{l}\text { Salinity, Heavy } \\
\text { metals } \\
\left(\mathrm{NH}_{4}^{+} \text {in aerat- }\right. \\
\text { ed-anaerobic) }\end{array}$ & $\begin{array}{l}\text { Two stage aero- } \\
\text { bic-anaerobic or } \\
\text { vice versa; Gas } \\
\text { collection; ex situ } \\
\text { treatment before } \\
\text { reinjection }\end{array}$ & $\begin{array}{l}\text { Air injection, } \\
\text { Leachate } \\
\text { recirculation }\end{array}$ & $\begin{array}{l}\text { Savings from } \\
\text { leachate } \\
\text { recirculation and } \\
\text { aeration, ex-situ } \\
\text { treatment cost if } \\
\text { present }\end{array}$ \\
\hline
\end{tabular}

* The costs are referred to the operational phase.

bioreactor type, both by reducing leachate treatment costs and by assuring sustainability requirements are achieved. Stabilization criteria of landfills is still a debated topic in the scientific literature (Barlaz et al., 2002; Laner et al., 2012; Stegmann et al. 2003; Valencia et al., 2009) since the criteria are not absolute and site specific conditions significant influence the values. In order to evaluate the sustainability achievement and aftercare completion, several approaches have been proposed such as the compliance with Final Storage Quality (FSQ) which defines the target emission values that must be achieved, impact risk assessment approaches, and performance based systems (Laner et al,. 2012). All of these approaches require a site-specific assessment in order to take into consideration the potential of natural attenuation or vulnerabilities (Barlaz et al., 2002; Laner et al., 2012; Rich et al., 2008).

In this study first order removal kinetics of organic and nitrogen concentration in leachate have been selected as criteria for the evaluation of the bioreactor stabilization performance (Ritzkowski et al., 2006) of the investigated lab-scale tests (Table 1).

A general overview of the stabilization capability associated with the different bioreactor types were calculated by the mean values of the COD and ammonia removal kinetics and standard deviations. The latter ones are represented as bar errors in Figure 2 in order to describe the distribution of values. Although there are variations in the operational management in the different investigated case studies, including the recirculation rate, waste composition, L/S ratio, air injection and experimental period (Table $1)$, the obtained mean COD kinetics can represent the general behaviour of each bioreactor type, as demonstrated by the standard deviations. The benefits of aerobic conditions are evident in the maximization of the COD removal with an average COD removal kinetic of $0.051 \mathrm{~d}^{-1}$. Hybrid and semi-aerobic bioreactor performances are between the
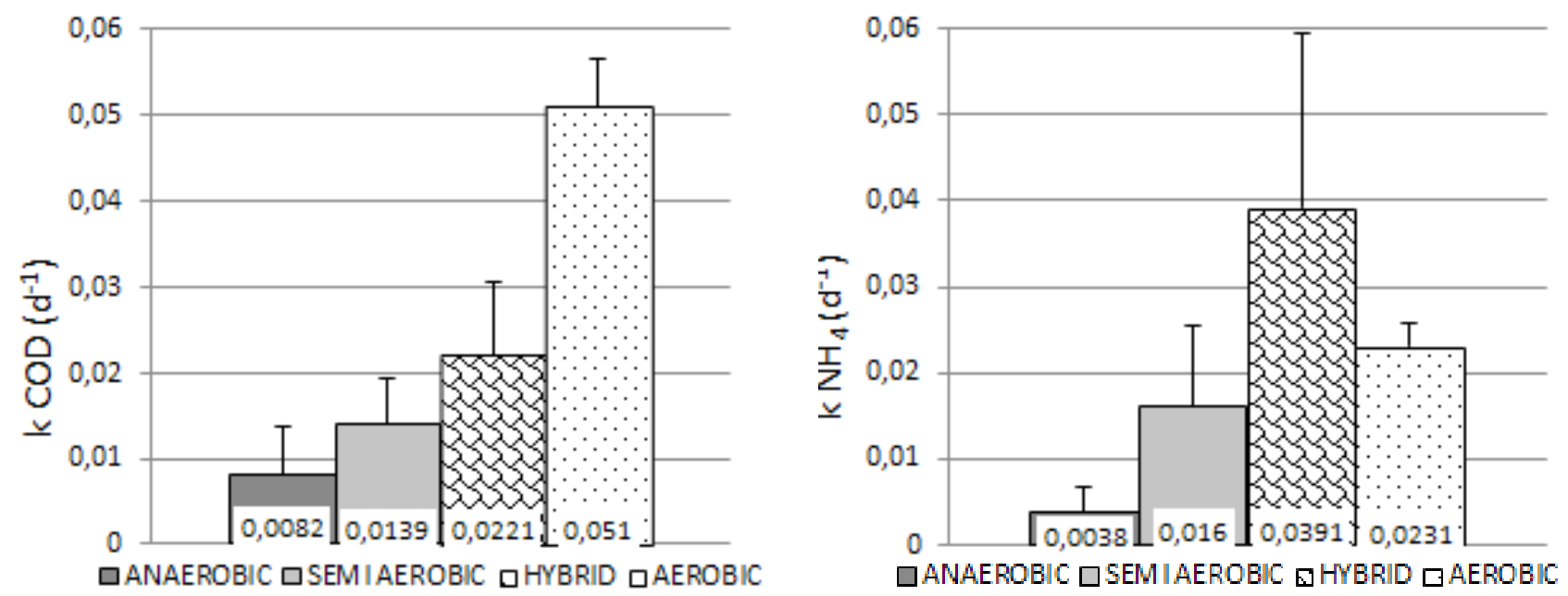

FIGURE 2: Mean values (numerically represented) and associated standard deviations (bar errors) of the COD and ammonia removal kinetics associate with each landfill bioreactor type starting from data collection and elaboration.(2012)). 
results for anaerobic and aerobic conditions presenting a $0.0221 d^{-1}$ and $0.0139 d^{-1}$ average removal kinetics, respectively. Different results were obtained for ammonia removal kinetics in which hybrid bioreactors demonstrated better average value compared to the other bioreactors $(0.0391$ $\mathrm{d}^{-1}$ ). The higher variability of the values around the mean makes these results carefully reliable since they are strongly influenced by the specific hybrid bioreactor application.

By the use of the mean COD and ammonia removal kinetics, it is possible to foresee and compare the stabilisation time for each bioreactor type. Considering the reference time $(\mathrm{T})$ required under aerobic conditions to achieve a 95\% contaminant removal (Figure 3), the time to achieve the same COD removal performance under hybrid, semi-aerobic, and anaerobic conditions increased by $2.3,3.7$, and 6.2-fold, respectively. In the case of ammonia removal, time is reduced by 0.7 -fold under hybrid conditions, while time increased by 1.7 and 3.7-fold under semi-aerobic and anaerobic conditions, respectively. According to these results, the faster the stabilization, the shorter the aftercare time and the lower the post closure care costs.

\section{CONCLUSIONS}

Anaerobic bioreactors improve, by leachate recirculation, the methane generation rate and the leachate quality compared to the traditional anaerobic landfills. However, ammonia accumulation and slow degradation kinetics remain the main challenges in anaerobic bioreactors compared to the others, putting anaerobic bioreactors far from sustainability requirements. Aerobic reactors increased the ammonia and COD average removal kinetics up to 6 times more than under strictly anaerobic conditions and reduced the time required to achieve a $95 \%$ removal of COD and ammonia by 6.2- and 3.7-fold, respectively. Aeration appears to be an effective alternative to the traditional anaerobic processes, although the need for forced ventilation systems, the complex operation and management, and the large energy consumption, with high operational and capital costs, make the aerated landfill not always technically and economically feasible. A semi-aerobic landfill achieves a performance between the anaerobic and aerobic bioreactors but lowering the typical operational costs of aerated landfills by removing the need for direct air injection. For this reason, the semi-aerobic system is recognized as a cost-effective, low technology landfill system. This system can also be feasibly implemented in developing countries, where financial constraints and limited technical knowledge are generally the main reasons for inadequate disposal. A limiting factor of aerobic bioreactors is the complete inhibition of the methane generation, making any energy recovery impossible. Hybrid bioreactors, which are operated under various combinations of aerobic and anaerobic conditions, achieve both energy recovery and/ or faster waste stabilization. In particular aerated-anaerobic hybrid reactors aim to enhance the biogas generation but this system will experience ammonia accumulation challenges, while facultative bioreactors combine both objectives which provides the best performance in terms of ammonia removal kinetics. In general, the best ammonia removal performance is achieved under hybrid conditions.

Due to the careful operation and construction requirements of bioreactor landfills, capital and operating costs would be greater compared to traditional landfills. However these costs will be recouped through future economy benefits from bioreactor landfills. In particular, the obtained results demonstrate the possibility of achieving shorter aftercare, reduced leachate treatment costs, reduced long term environmental risks, and an earlier reuse of the land. Detailed analysis of costs related to full-scale bioreactors is still a crucial aspect to be further investigated.

Moreover, the transfer from a lab-scale to full-scale bioreactor still remains a significant issue to be explored since much higher benefits are achieved under lab-scale investigation rather than at full-scale application due to the challenges with reproducing optimum and homogeneous conditions. However, knowing the general behavior of each bioreactor type at lab scale allows the identification of the best bioreactor solution at a larger scale according to the site specific objectives and in-situ conditions.

\section{REFERENCES}

Ahmadifar, M., Sartaj, M., Abdallah, M., 2016. Investigating the performance of aerobic, semi-aerobic, and anaerobic bioreactor landfills for MSW management in developing countries. J. Mater. Cycles Waste Manag. 18, 703-714. doi:10.1007/s10163-015-0372-0

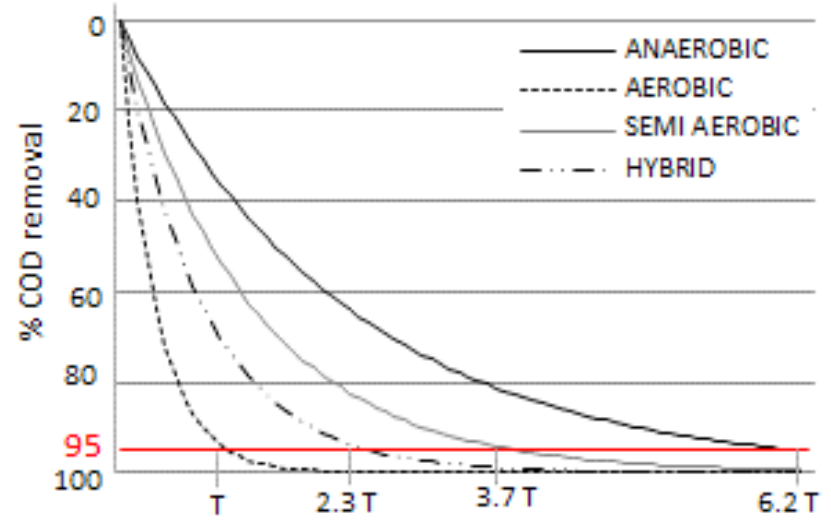

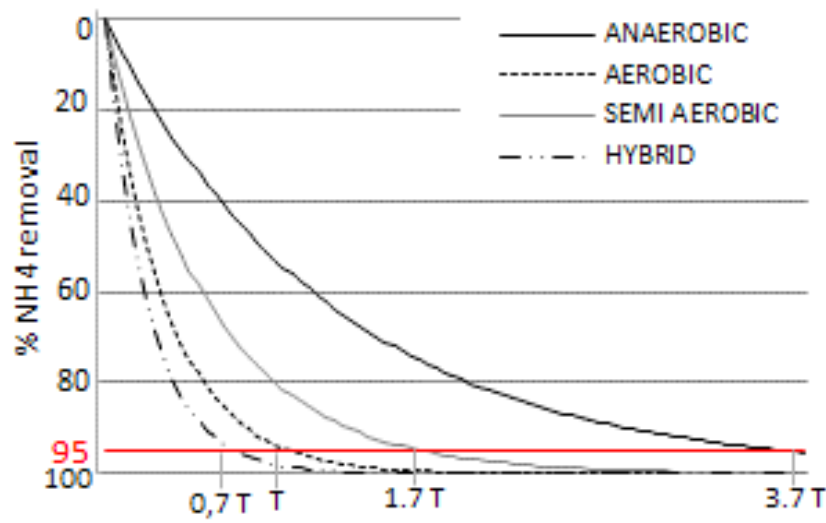

FIGURE 3: Variation of the percentage removal of COD and ammonia over time, according to the mean obtained removal kinetics. Reference time $(\mathrm{T})$ is the time required to achieve a $95 \%$ removal under aerobic conditions. 
Anex, R.P. (1996) Optimal Waste Decomposition- Landfill as Treatment Process. Journal of Environmental Engineering, ASCE, 122(11) 964-974.

Barlaz, M.A., Rooker, A.P., Kjeldsen, P., Gabr, M.A., Borden, R.C., 2002. Critical Evaluation of Factors Required To Terminate the Postclosure Monitoring Period at Solid Waste Landfills. Environ. Sci. Technol. 36, 3457-3464.

Berge, N.D., Reinhart, D.R., Batarseh, E.S., 2009. An assessment of bioreactor landfill costs and benefits. Waste Manag. 29, 1558-1567. doi:10.1016/j.wasman.2008.12.010

Berge, N.D., Reinhart, D.R., Dietz, J., Townsend, T., 2006. In situ ammonia removal in bioreactor landfill leachate. Waste Manag. 26, 334-343. doi:10.1016/j.wasman.2005.11.003

Berge, N.D., Reinhart, D.R., Townsend, T.G., 2005. The Fate of Nitrogen in Bioreactor Landfills. Crit. Rev. Environ. Sci. Technol. 35, 365399. doi:10.1080/10643380590945003

Bolyard, S.C., Reinhart, D.R., 2015. Application of landfill treatment approaches for stabilization of municipal solid waste. Waste Manag. doi:10.1016/j.wasman.2016.01.024

Borglin, S.E., Hazen, T.C., Oldenburg, C.M., Zawislanski, P.T., 2004. Comparison of aerobic and anaerobic biotreatment of municipal solid waste. J. Air Waste Manag. Assoc. doi:10.1080/10473289.2004 10470951

Christensen, T.H., Manfredi, S., Knox, K, 2011. Landfilling: Reactor Landfills. In: Christensen, T.H. (Ed.), Solid Waste Technology and Management, vol. 2. Blackwell Publishing Ltd.

Cossu, R., 2012. The environmentally sustainable geological repository: The modern role of landfilling. Waste Manag. 32, 243-244. doi:10.1016/j.wasman.2011.11.005

Cossu, R., 2010. Technical evolution of landfilling. Waste Manag. 30 947-948. doi:10.1016/j.wasman.2010.03.003

Cossu, R., Morello, L., Raga, R., Cerminara, G., 2016. Biogas production enhancement using semi-aerobic pre-aeration in a hybrid bioreactor landfill. Waste Manag. 55, 83-92. doi:10.1016/j.wasman.2015.10.025

Cossu, R., Raga, R., Rossetti, D., 2003. The PAF model: An integrated approach for landfill sustainability. Waste Manag. 23, 37-44. doi:10.1016/S0956-053X(02)00147-2

De Abreu, R., La Motta, E., McManis, K., 2005. Facultative landfill bioreactors (FLB): results of a pilot-scale study. Waste Containment Remediat., 1-13.

EPA, 2018a. Bioreactors. https://archive.epa.gov/epawaste/nonhaz/ municipal/web/html/bioreactors.html. (Accessed on 5th March 2018)

EPA, 2018b. Global Mitigation of Non-CO2 Greenhouse Gases: Landfills. https://www.epa.gov/global-mitigation-non-co2-greenhouse-gases/global-mitigation-non-co2-greenhouse-gases-landfills (accessed on 5th August 2018)

Erses, A.S., Onay, T.T., Yenigun, O., 2008. Comparison of aerobic and anaerobic degradation of municipal solid waste in bioreactor landfills. Bioresour. Technol. 99, 5418-5426. doi:10.1016/j. biortech.2007.11.008

EU, Proposal of a Directive of the European Parliament and of the Council, 2015. http://eur-lex.europa.eu/legal-content/EN/TXT/?uri=CELEX:52015PC0595

Fellner, J., Döberl, G., Allgaier, G., Brunner, P.H., 2009. Comparing field investigations with laboratory models to predict landfill leachate emissions. Waste Manag. 29, 1844-1851. doi:10.1016/j.wasman.2008.12.022

Filipkowska, U., 2008. Effect of recirculation method on quality of landfill leachate and effectiveness of biogas production. Polish J. Environ. Stud. 17, 199-207.

Giannis, A., Makripodis, G., Simantiraki, F., Somara, M., Gidarakos, E., 2008. Monitoring operational and leachate characteristics of an aerobic simulated landfill bioreactor. Waste Manag. 28, 13461354. doi:10.1016/j.wasman.2007.06.024

Hanashima, M., Yamasaki, K., Kuroki, T., Onishi, K., 1981. Heat and gas flow analysis in semiaerobic landfill. J. Environ. Eng. Divis., Proc. Am. Soc. Civ. Eng. 107 (EE1), 1-9.

Hater, G.R., Young, K.E., Simpson, C., Harris, J.M., 2001. Economics of Eight Scenarios for Landfill Bioreactors as Compared to a Base Case Subtitle D Landfill. Waste Tech 2001, San Diego, CA, February.

Heimovaara, T.J. Cossu, R, van der Sloot, H.A , 2014. State of the art and perspectives for sustainable landfills. In: Cossu, R., van der Sloot, H.A. (Eds.), Sustainable Landfilling. CISA Publisher, ISBN 978-88-6265-005-2.
He, P., Yang, N., Gu, H., Zhang, H., Shao, L., 2011. N2O and NH3 emissions from a bioreactor landfill operated under limited aerobic degradation conditions. J. Environ. Sci. 23, 1011-1019. doi:10.1016/ S1001-0742(10)60574-8

He, R., Liu, X. wen, Zhang, Z. jian, Shen, D. sheng, 2007. Characteristics of the bioreactor landfill system using an anaerobic-aerobic process for nitrogen removal. Bioresour. Technol. 98, 2526-2532. doi:10.1016/j.biortech.2006.09.013

He, Y., Guo, L., Gao, J., 2012. The Advantages and Mechanism of Semi-aerobic Landfill. Energy Procedia 17, 391-396. doi:10.1016/j. egypro.2012.02.111

Hirata, O., Matsufuji, Y., Tachifuji, A., Yanase, R., 2012. Waste stabilization mechanism by a recirculatory semi-aerobic landfill with the aeration system. J. Mater. Cycles Waste Manag. 14, 47-51. doi:10.1007/s10163-011-0036-7

Hrad, M., Gamperling, O., Huber-Humer, M., 2013. Comparison between lab- and full-scale applications of in situ aeration of an old landfill and assessment of long-term emission development after completion. Waste Manag. 33, 2061-2073. doi:10.1016/j.wasman.2013.01.027

HUANG, Q., YANG, Y., PANG, X., WANG, Q., 2008. Evolution on qualities of leachate and landfill gas in the semi-aerobic landfill. J. Environ. Sci. 20, 499-504. doi:10.1016/S1001-0742(08)62086-0

Huo, S.L., Xi, B.D., Yu, H.C., Fan, S.L., Jing, S., Liu, H.L., 2008. A laboratory simulation of in situ leachate treatment in semi-aerobic bioreactor landfill. Water SA 34, 133-140.

Hupe, K., Heyer, K. and Stegmann, R., 2003: Water infiltration for enhanced in situ stabilization. In: Proceedings Sardinia 2003, Ninth International Waste Management and Landfill Symposium, 6-10 October, Sardinia, Italy. Proceedings. CD-ROM, CISA - Environmental Sanitary Engineering Centre, Cagliari, Italy.

IPPC, 2006. IPPC Guidelines for National Greenhouse Gas Inventories. Volume 5: Waste. Chapter 3: Solid Waste Disposal. https://www. ipcc-nggip.iges.or.jp/public/2006gl/vol5.html (accessed 5 August 2018)

Jacobs, J., Scharff, H., Van Arkel, F., De Gier, C.W., 2003. Odour reduction by aeration of landfills: experience, operation and costs. In: Conference Proceedings, 9th International Waste Management and Landfill Symposium, Cagliari, Italy.

Jeong, S., Nam, A., Yi, S.M., Kim, J.Y., 2015. Field assessment of semi-aerobic condition and the methane correction factor for the semi-aerobic landfills provided by IPCC guidelines. Waste Manag. 36, 197-203. doi:10.1016/j.wasman.2014.10.020

JICA, 2004. Detailed design for development of semi-aerobic system pilot project at Sisdole landfill site (vol. I), Lalitpur, Nepal: East Consult. (P.) Ltd.

Jokela, J.P.Y, Kettunen, R. H Sormunen, K.M Rintala, J.A., 2002. Biological nitrogen removal from municipal landfill leachate: Low-cost nitrification in biofilters and laboratory scale in-situ denitrification. Water Res. 36, 4079-4087. doi:10.1016/S0043-1354(02)00129-X

Karnik, M. \& C. Perry (1997) Cost Implications of Operating Landfills as Flushing Bioreactors. Proceedings from Sardinia 97, Sixth International Landfill Symposium, Volume I, S. Margherita di Pula, Cagliari, Italy, 13-17 October, 1997, Volume I, 419-426

Khalil, K., Mary, B., Renault, P., 2004. Nitrous oxide production by nitrification and denitrification in soil aggregates as affected by 02concentration. Soil Biol. Biochem. 36, 687-699. doi:10.1016/j. soilbio.2004.01.004Kim H., 2005. Comparative studies of aerobic and anaerobic landfills using simulated landfill lysimeters. Doctoral dissertation. Retrieved from University of Florida Digital Collections. http://ufdc.ufl.edu//AA00025781/00001

Kim, H., Jang, Y.C., Townsend, T., 2011. The behavior and long-term fate of metals in simulated landfill bioreactors under aerobic and anaerobic conditions. J. Hazard. Mater. 194, 369-377. doi:10.1016/j. jhazmat.2011.07.119

Kylefors, K., Andreas, L., Lagerkvist, A., 2003. A comparsion of smallscale, pilot scale and large -scale tests for predicting leaching behavioud of landfilled wastes. Waste Manag. 23, 45-59.

Laner, D., Crest, M., Scharff, H., Morris, J.W.F., Barlaz, M.A., 2012. A review of approaches for the long-term management of municipal solid waste landfills. Waste Manag. 32, 498-512. doi:10.1016/j. wasman.2011.11.010

Lavagnolo MC, Grossule V, Raga R. Innovative dual-step management of semi-aerobic landfill in a tropical climate. Waste Manag [Internet]. 2018;1-10. Available from: https://doi.org/10.1016/j.wasman.2018.01.017 
Long, Y., Hu, L. fang, Shen, D.S., 2009a. Nitrogen transformation in the hybrid bioreactor landfill. Bioresour. Technol. 100, 2527-2533. doi:10.1016/j.biortech.2008.11.036

Long, Y., Long, Y.Y., Liu, H.C., Shen, D.S., 2009b. Degradation of refuse in hybrid bioreactor landfill. Biomed. Environ. Sci. 22, 303-310. doi:10.1016/S0895-3988(09)60060-X

Mali Sandip, T., Khare Kanchan, C., Biradar Ashok, H., 2012. Enhancement of methane production and bio-stabilisation of municipal solid waste in anaerobic bioreactor landfill. Bioresour. Technol. 110, 10-17. doi:10.1016/j.biortech.2011.12.027

Matsufuji, Y., Kobayashi, H., Tanaka, A., Ando, S., Kawabata, T., Hanashima, M., 1996. Generation of greenhouse effect gases by different landfill types and methane gas control. In: Proceedings of 7th ISWA International Congress and Exhibition, Yokohama, Japan, pp. 230-237.

Mertoglu, B., Calli, B., Inanc, B., Ozturk, I., 2006. Evaluation of in situ ammonia removal in an aerated landfill bioreactor. Process Biochem. 41, 2359-2366. doi:10.1016/j.procbio.2006.06.014

Ministry of the Environment (Japan), 2018. http://www.env.go.jp/recycle/circul/venous_industry/index.html (Accessed on 6th June 2018)

Morello, L., Raga, R., Lavagnolo, M.C., Pivato, A., Ali, M., Yue, D., Cossu, R., 2017. The S.An.A.® concept: Semi-aerobic, Anaerobic, Aerated bioreactor landfill. Waste Manag. doi:10.1016/j.wasman.2017.05.006

Mummey, D.L., Smith, J.L., Bolton, H., 1994. Nitrous oxide flux from a shrub-steppe ecosystem: Sources and regulation. Soil Biol. Biochem. 26, 279-286. doi:10.1016/0038-0717(94)90168-6

Nikolaou, A., Giannis, A., Gidarakos, E., 2009. Comparison of aerobic and anaerobic degradation of municipal solid wastes in lab scale bioreactors. Proceedings of the 11th International Conference on Environmental Science and Technology. Chaina, Crete, Greece, 3-5 September 2009

Omar, H., Rohani, S., 2015. Treatment of landfill waste, leachate and landfill gas: A review. Front. Chem. Sci. Eng. doi:10.1007/s11705015-1501-y

Price, G.A., Barlaz, M.A., Hater, G.R., 2003. Nitrogen management in bioreactor landfills. Waste Manag. 23, 675-688. doi:10.1016/ S0956-053X(03)00104-1

Powell, J., Jain, P., Kim, H., Townsend, T., Reinhart, D., 2006. Changes in landfill gas quality as a result of controlled air injection. Environ. Sci. Technol. 40, 1029-1034. doi:10.1021/es051114j

Price, G.A., Barlaz, M.A., Hater, G.R., 2003. Nitrogen management in bioreactor landfills. Waste Manag. 23, 675-688. doi:10.1016/ S0956-053X(03)00104-1

Purcell, B.E., Sollars, C.J., Butler, A.P., 1997. Enhanced moisture movement in simulated landfill environments. In: Proceedings Sardinia 97, Sixth International Landfill Symposium, Vol. I, CISA, Cagliari, pp. 409-418.

Raga, R., Cossu, R., 2014. Landfill aeration in the framework of a reclamation project in Northern Italy. Waste Manag. 34, 683-691. doi:10.1016/j.wasman.2013.12.011

Read, A.D., Hudgins, M., Phillips, P., 2001. Aerobic landfill test cells and their implications for sustainable waste disposal. Geogr. J. 167, 235-247. doi:10.1111/1475-4959.00021

Reinhart, D.R., Mccreanor, P.T., Townsend, T., 2002. Waste Management \& Research The bioreactor landfill : Its status and future. Waste Manag. Res. 172-186. doi:10.1177/0734242X0202000209

Rich, C., Gronow, J., Voulvoulis, N., 2008. The potential for aeration of MSW landfills to accelerate completion. Waste Manag. 28, 10391048. doi:10.1016/j.wasman.2007.03.022

Ritzkowski, M., 2011. How Does Landfill Aeration Impact on Leachate Composition? Proceedings Sardinia 2011, Thirteenth International Waste Management and Landfill Symposium S. Margherita di Pula, Cagliari, Italy; 3 - 7 October 2011

Ritzkowski, M., Heyer, K.U., Stegmann, R., 2006. Fundamental processes and implications during in situ aeration of old landfills. Waste Manag. 26, 356-372. doi:10.1016/j.wasman.2005.11.009

Ritzkowski, M., Stegmann, R., 2013. Landfill aeration within the scope of post-closure care and its completion. Waste Manag. 33, 20742082. doi:10.1016/j.wasman.2013.02.004

Ritzkowski, M., Stegmann, R., 2012. Landfill aeration worldwide: Concepts, indications and findings. Waste Manag. 32, 1411-1419. doi:10.1016/j.wasman.2012.02.020

Ritzkowski, M., Stegmann, R., 2007. Controlling greenhouse gas emissions through landfill in situ aeration. Int. J. Greenh. Gas Control 1, 281-288. doi:10.1016/S1750-5836(07)00029-1
Ritzkowski, M., Stegmann, R., 2005. Mechanisms Affecting the Leachate Quality in the Course of Landfill in Situ Aeration. Proceedings Sardinia 2005, Tenth International Waste Management and Landfill Symposium. S. Margherita di Pula, Cagliari, Italy; 3 - 7 October 2005

Ritzkowski, M., Stegmann, R., 2003. Emission behaviour of aerated landfills: results of laboratory scale investigations. Proceedings Sardinia 2003, Ninth International Waste Management and Landfill Symposium. S. Margherita di Pula, Cagliari, Italy; 6 - 10 October 2003

Šan, I., Onay, T.T., 2001. Impact of various leachate recirculation regimes on municipal solid waste degradation. J. Hazard. Mater. 87, 259-271. doi:10.1016/S0304-3894(01)00290-4

Sanphoti, N., Towprayoon, S., Chaiprasert, P., Nopharatana, A., 2006 The effects of leachate recirculation with supplemental water addition on methane production and waste decomposition in a simulated tropical landfill. J. Environ. Manage. 81, 27-35. doi:10.1016/j. jenvman.2005.10.015

Shao, L.M., He, P.J., Li, G.J., 2008. In situ nitrogen removal from leachate by bioreactor landfill with limited aeration. Waste Manag. 28, 1000-1007. doi:10.1016/j.wasman.2007.02.028

Shimaoka T., Matsufuji Y., Hanashima M., 2000. Characteristic and Mechanism of Semi-Aerobic Landfill on Stabilization of Solid Waste. Intercontinental Landfill Research Symposium, 2000.

Shou-liang, H., Bei-dou, X., Hai-chan, Y., Shi-lei, F., Jing, S., Hong-liang, L. 2008. In situ simultaneous organics and nitrogen removal from recycled landfill leachate using an anaerobic-aerobic process. Bioresour. Technol. 99, 6456-6463. doi:10.1016/j.biortech.2007.11.047

Sinan Bilgili, M., Demir, A., Ince, M., Özkaya, B., 2007. Metal concentrations of simulated aerobic and anaerobic pilot scale landfill reactors. J. Hazard. Mater. 145, 186-194. doi:10.1016/j. jhazmat.2006.11.014

Slezak, R., Krzystek, L., Ledakowicz, S., 2015. Degradation of municipal solid waste in simulated landfill bioreactors under aerobic conditions. Waste Manag. 43. doi:10.1016/j.wasman.2015.06.017

Slezak, R., Krzystek, L., Ledakowicz, S., 2010. Simulation of aerobic landfill in laboratory scale lysimeters - effect of aeration rate. Chem. Pap. 64, 223-229. doi:10.2478/s11696-009-0113-8

Stegmann, R., Heyer, K.U., Hupe, K., Ritzkowski, M., 2003. Discussion of criteria for the completion of landfill aftercare. Paper presented at the Sardinia International Waste Management and Landfill Symposium, Sardinia, Italy.

Stessel, R.I., Murphy, R.J., 1992. A lysimeter study of the aerobic landfill concept. Waste Manag. Res. 10, 485-503. doi:10.1016/0734 242X(92)90089-4

Sun, F., Sun, B., Li, Q., Deng, X., Hu, J., Wu, W., 2014. Pilot-scale nitrogen removal from leachate by ex situ nitrification and in situ denitrification in a landfill bioreactor. Chemosphere 101. doi:10.1016/j. chemosphere.2013.12.030

Sun, X., Zhang, H., Cheng, Z., 2017. Use of bioreactor landfill for nitrogen removal to enhance methane production through ex situ simultaneous nitrification-denitrification and in situ denitrification. Waste Manag. 66, 97-102. doi:10.1016/j.wasman.2017.04.020

Sun, Y., Wang, Y.N., Sun, X., Wu, H., Zhang, H., 2013. Production characteristics of $\mathrm{N} 2 \mathrm{O}$ during stabilization of municipal solid waste in an intermittent aerated semi-aerobic bioreactor landfill. Waste Manag. 33, 2729-2736. doi:10.1016/j.wasman.2013.08.013

Sutthasil, N., Chiemchaisri, C., Chiemchaisri, W., Wangyao, K., Tawprayoon, S., Endo, K., Yamada, M., 2014. Comparison of Solid Waste Stabilization and Methane Emission from Aerobic and Semi-aerobic Landfills Operated in Tropical Conditions. Environ. Eng. Res. 19, 261-268.

Theng, L.C., Matsufuji, Y., Mohd, N.H., 2005. Implementation of the semi-aerobic landfill system (Fukuoka method) in developing countries: A Malaysia cost analysis. Waste Manag. 25, 702-711. doi:10.1016/j.wasman.2005.01.008

Tsujimoto, Y., Masuda, J., Fukuyama, J., Ito, H., 1994. N2o Emissions at Solid-Waste Disposal Sites in Osaka City. J. Air Waste Manage. Assoc. 44, 1313-1314. doi:10.1080/10473289.1994.10467327

US6398958 B1 2002. Gary R. Hater, Roger B. Green, Jeffrey M. Harris. Patent Facultative Landfill Bioreactor, Waste Management. Inc

Valencia, R., van der Zon, W., Woelders, H., Lubberding, H.J., Gijzen, H.J., 2009. Achieving "Final Storage Quality" of municipal solid waste in pilot scale bioreactor landfills. Waste Manag. 29, 78-85. doi:10.1016/j.wasman.2008.02.008 
Valencia, R., Zon, W. van der, Woelders, H., Lubberding, H.J., Gijzen, H.J., 2011. Anammox: An option for ammonium removal in bioreactor landfills. Waste Manag. 31, 2287-2293. doi:10.1016/j.wasman.2011.06.012

Venterea, R.T., Rolston, D.E., 2000. Mechanisms and kinetics of nitric and nitrous oxide production during nitri $₫$ cation in agricultural soil. Glob. Chang. Biol. 6, 303-316.

Vigneron, V., Ponthieu, M., Barina, G., Audic, J.M., Duquennoi, C., Mazéas, L., Bernet, N., Bouchez, T., 2007. Nitrate and nitrite injection during municipal solid waste anaerobic biodegradation. Waste Manag. 27, 778-791. doi:10.1016/j.wasman.2006.02.020

Walker, A.N., Beaven, R.P., Powrie, W., 1997. Overcoming problems in the development of a high rate flushing bioreactor landfill. In: Proceedings Sardinia 97, Sixth International Landfill Symposium, Vol. I, CISA, Cagliari, pp. 397-408.

Warith, M., 2002. Bioreactor landfills: Experimental and field results. Waste Manag. 22, 7-17. doi:10.1016/S0956-053X(01)00014-9

Watzinger, a, Reichenauer, T.G., Blum, W.E.H., Gerzabek, M.H., Zechmeister-Boltenstern, S., 2005. The Effect of Landfill Leachate Irrigation on Soil Gas Composition: Methane Oxidation and Nitrous Oxide Formation. Water, Air, Soil Pollut. 164, 295-313.

Wu, C., Shimaoka, T., Nakayama, H., Komiya, T., Chai, X., Hao, Y., 2014. Influence of aeration modes on leachate characteristic of landfills that adopt the aerobic-anaerobic landfill method. Waste Manag. 34, 101-111. doi:10.1016/j.wasman.2013.10.015
Xu, Q., Jin, X., Ma, Z., Tao, H., Ko, J.H., 2014. Methane production in simulated hybrid bioreactor landfill. Bioresour. Technol. 168, 92-96. doi:10.1016/j.biortech.2014.03.036

Xu, Q., Tian, Y., Wang, S., Ko, J.H., 2015. A comparative study of leachate quality and biogas generation in simulated anaerobic and hybrid bioreactors. Waste Manag. 41, 94-100. doi:10.1016/j.wasman.2015.03.023

Yang, Y., Yue, B., Yang, Y., Huang, Q., 2012. Influence of semi-aerobic and anaerobic landfill operation with leachate recirculation on stabilization processes. Waste Manag. Res. 30, 255-265. doi:10.1177/0734242X11413328

Yuen, S., Styles, J., Wang, Q., McMahon, T., 1999. Findings from a fullscale bioreactor landfill study in Australia. Proceedings Sardinia '99, 7th International Waste management and Landfill Symposium, Environmentla Sanitary Engineering Center (CISA), Cagiari, Italy.

Zeng, X.L., Ding, W.C., Long, T.R., Zhang, L.L., Lin, C.B., 2006. Influence of landfill structures on stabilization of fully recycled leachate. J. Cent. South Univ. Technol. (English Ed. 13, 321-324. doi:10.1007/ s11771-006-0133-2

Zhong, Q., Li, D., Tao, Y., Wang, X., He, X., Zhang, J., Zhang, J., Guo, W., Wang, L., 2009. Nitrogen removal from landfill leachate via ex situ nitrification and sequential in situ denitrification. Waste Manag. 29, 1347-1353. doi:10.1016/j.wasman.2008.10.014 